\title{
Imidazolium-based ionic liquids as co-surfactants in aqueous micellar two- phase systems composed of nonionic surfactants and their aptitude for recovery of natural colorants from fermented broth
}

\author{
Fábio Aurélio Esteves Torres, Ana Clara de Almeida Francisco, Jorge Fernando Brandão Pereira, \\ Valéria de Carvalho Santos-Ebinuma* \\ Universidade Estadual Paulista (UNESP), School of Pharmaceutical Sciences, Campus (Araraquara), Department of Bioprocess and Biotechnology, Araraquara, SP, Brazil
}

\begin{abstract}
A B S T R A C T
Over the last few years, synthetic colorants have been increasingly replaced by natural alternatives that cause fewer adverse health effects and can show biological activity. These biocompounds can be produced by fermentative processes using microorganisms such as bacteria and filamentous fungi. However, the biocompounds must be extracted from the fermented broth before industrial application. Thus, the development of effective techniques for the extraction and purification of biocolorants with high recovery is of great interest. The main goal of this study was to evaluate the recovery of natural colorants produced by Talaromyces amestolkiae using aqueous micellar two-phase system (AMTPS) composed of Triton X-114 and Ionic Liquids (ILs) from the imidazolium family as co-surfactants. Preliminary experiments were performed to determine the binodal curves in both presence and absence of fermented broth containing the red colorants. Subsequently, a set of partitioning experiments was performed using the developed systems. Under all conditions, the red colorants were partitioned mainly into the micelle-rich phase. The systems with low concentrations of Triton X-114 and ILs showed high partition coefficients. The system $\left(0.1 \mathrm{wt} \%\left[\mathrm{C}_{10} \mathrm{mim}\right] \mathrm{Cl}+3 \mathrm{wt} \%\right.$ Triton $\left.\mathrm{X}-114\right)$ with the highest partition coefficient $\left(K_{R C}=6.12\right)$ was used to study the effect of temperature on partitioning. The partitioning of the red colorants was strongly influenced by the temperature, and $K_{R C}$ increased to 24.71 at $45^{\circ} \mathrm{C}$. The results of this study demonstrate that the partitioning of biomolecules can be controlled by the presence of ILs, mainly by electrostatic interactions, or by the proper adjustment of the partitioning temperature.
\end{abstract}

\section{Introduction}

With the increasing demand for eco-friendly/non-toxic colorants [1-3], there has been an increasing trend towards replacing synthetic colorants by natural alternatives because the latter cause fewer adverse health effects and have interesting biological properties, such as antioxidant and anticancer activity. Natural colorants can be obtained from plants, insects, microorganisms, animals, and minerals [4,5]. A wide range of colorants has been produced through fermentative processes by filamentous fungi, including carotenoids, melanin, flavins, phenazine, quinones, and violacein [6]. Fungi from Talaromyces species produce red colorants that are usually composed of an azaphilone ring (e.g., monascorubrin, rubropunctatin, and monascorubramine). These colorants have potential for industrial application because they are nontoxic and more stable than other natural congeners [7].

Despite their interesting features and industrial importance, natural colorants derived from microorganisms are, in most cases, complex mixtures with variable compositions [8]. Thus, the selective extraction of these compounds from contaminants (such as proteins, sugars, salts) present in the fermented broth is required for their industrial application. The conventional downstream processing of natural products, in particular, their recovery and separation, is hampered by several major drawbacks, such as low recovery yield, low selectivity, time-intensive extraction processes, and the need for extensive heating and mixing [9]. Among the conventional downstream techniques used in the recovery of biomolecules, liquid-liquid extraction using organic solvents is the classical method, followed by chromatographic techniques [10]. However, as is widely known in industry, the extensive use of organic solvents has certain limitations because of the toxicity and environmental impact of these solvents besides their low biocompatibility, and the consequent degradation of metabolites, whereas the chromatographic techniques are expensive [11].

\footnotetext{
* Corresponding author.

E-mail address: valeriac@fcfar.unesp.br (V.d.C. Santos-Ebinuma).
} 
The use of aqueous two-phase systems (ATPS) as alternative liquidliquid extraction systems for biomolecules has been proposed [12]. These systems form two immiscible aqueous-rich phases by the combination, at least, of two structurally different compounds (polymers, salts, surfactants, sugars, or Ionic Liquids (ILs)) above certain concentrations [13]. Since both coexisting phases of ATPS are mainly composed of water, such systems are excellent for maintaining the native conformation and biological properties of biomolecules. There are many possible combinations of phase-forming agents as effective platforms for improving the extraction and selectivity yields of several biocompounds $[14,15]$. Aqueous micellar two-phase systems (AMTPS) composed of surfactants are among the most interesting of these systems. Surfactants are organic molecules that contain both a polar and a non-polar group [13]. In aqueous solution, when the surfactant concentration exceeds the critical micelle concentration (CMC), the surfactant molecules form dynamic aggregates called micelles [16]. With increasing temperature, the micelle clusters and intermicellar attractions result in turbidity (a cloudy region) and the spontaneous formation of two immiscible phases, i.e., a micelle-rich phase and a micellepoor phase $[17,18]$. The temperature at which phase separation occurs is generally known as the cloud point temperature $\left(T_{c p}\right)$, and this value depends on the surfactant structure and concentration, as well as the presence of additives [19]. This process is reversible if the system is cooled below its $T_{c p}$ [20].

When AMTPS are generated by mixing two different types of surfactants (generally a nonionic and an ionic surfactant), mixed micelles can be formed. The formation of mixed micelles affects the phase separation behavior of the systems, generally by increasing the $T_{c p}$. This separation occurs because of competition between the internal energy effects, which causes the separation of the micelles from the water, and entropic effects, which promote the miscibility of the micelles with water [21]. The use of additives or ionic surfactants in these systems may modify both the intermicellar and intramicellar interactions because of electrostatic screening [22]. As a result, the presence of ionic surfactants promotes the formation of charged, mixed micelles that could improve the recovery of several biomolecules.

The influence of different salts on the $T_{c p}$ has been well described in the literature, and, although several salts reduce the $T_{c p}$, others increase it [19] by changing the intramicellar and intermicellar interactions. On the other hand, the changes in the interactions are not exclusively induced by the addition of salts but can be obtained by the formation of mixed micellar systems, which can also affect the interactions, resulting in different cloud points. In contrast to the effects of inorganic salts, which have been widely discussed in the literature $[19,23,24]$, the use of different ILs and their influence on the binodal curves of AMTPS has been relatively underexplored.

By common definition, ILs are salts with low melting points that are typically composed of organic or inorganic anions and an organic cation associated with one or more alkyl chain substituents [25,27]. Because of the many ion arrangements, these compounds have unique characteristics, such as negligible vapor pressures, high thermal and chemical stabilities, high solvation capacities, and variable viscosities and densities [26]. Some ILs containing long alkyl chains exhibit surfactant characteristics [28] and can be used to form AMTPS or can be added as co-surfactants in combination with nonionic surfactants to form mixed micelles, thereby improving the partitioning selectivity of biomolecules. As an example, in contrast to other salts, imidazolium-based ILs have a pre-organized structure, which is mainly based on hydrogen bonds, in the liquid state. These assemblies are described as "supramolecular" fluids $[29,30]$. Depending on the chain length, ILs from the imidazolium family exhibit amphiphilic characteristics and can induce the same aggregation behavior as traditional cationic surfactants [31].

In the first stage of this study, the binodal curves of the AMTPS were obtained. Above the $T_{c p}$, two phases are formed; thus, it is essential to determine the temperature at which phase separation occurs prior to performing any extraction or separation processes. As mentioned, only a few reports have documented the binodal curves of AMTPS in the presence of ILs. Recently, Vicente et al. [13] studied the effect of ILs from the imidazolium, phosphonium, and quaternary ammonium families and determined the respective binodal curves with Triton X-114 in Mcllvaine buffer at $\mathrm{pH}$ 7.0. The previous work of these authors has encouraged us to carry out a series of experiments for the extraction of colorants directly from fermented broth using $\left[\mathrm{C}_{x} \mathrm{mim}\right] \mathrm{Cl}$-based AMTPS. However, when the systems composed of $\left[\mathrm{C}_{10} \mathrm{mim}\right] \mathrm{Cl}$ and $\left[\mathrm{C}_{12} \mathrm{mim}\right] \mathrm{Cl}$ were used as co-surfactants, a two-phase region was not attained probably due to the presence of fermented broth which is a complex medium that can affect the phase equilibrium, and consequently, affecting the binodal curve.

Considering the interesting features of AMTPS and imidazoliumbased ILs, herein, we aim to understand the influence of the chain length of the cation of imidazolium-based ILs on the phase separation of AMTPS containing the nonionic surfactant Triton X-114 and the efficacy of these systems for the recovery of red colorants from the fermented broth of Talaromyces amestolkiae.

\section{Materials and methods}

\subsection{Materials}

The imidazolium-based ILs used in this work, 1-dodecyl-3-methylimidazolium chloride $\left(\left[\mathrm{C}_{12} \mathrm{mim}\right] \mathrm{Cl}\right)$ (purity $\left.99 \%\right)$, 1-decyl-3-methylimidazolium chloride $\left(\left[\mathrm{C}_{10} \mathrm{mim}\right] \mathrm{Cl}\right)$ (purity $\left.99 \%\right)$, and 1-methyl-3-octylimidazolium chloride $\left(\left[\mathrm{C}_{8} \mathrm{mim}\right] \mathrm{Cl}\right)$ (purity $>98 \%$ ), were acquired from Ionic Liquids Technologies (IoLiTec, Heilbronn, Germany). The<smiles>CCCCCCCCCCCCn1cc[n+](C)c1</smiles>
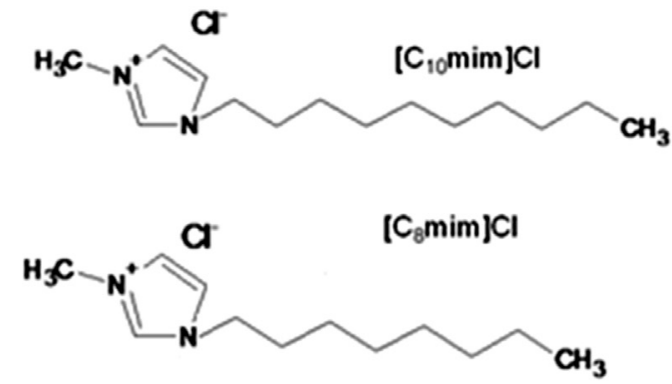

Triton X-114

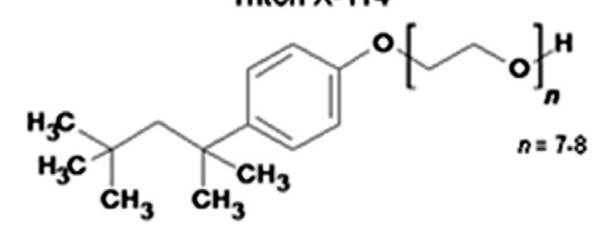

Fig. 1. Chemical structure representation of the ILs and Triton X-114 used in the present work. 
surfactant, (1,1,3,3-tetramethylbutyl)phenyl-polyethylene glycol (Triton X-114), was purchased from Sigma-Aldrich ${ }^{\circledast}$ (St. Louis, MO) (Fig. 1). All other reagents were of analytical grade and were used as received unless otherwise stated. All solutions were prepared in Mcllvaine buffer at $\mathrm{pH} 6.5$, which comprises $2.64 \mathrm{mmol} \mathrm{dm}^{-3}$ disodium phosphate and $0.68 \mathrm{mmol} \mathrm{dm}{ }^{-3}$ citric acid in water purified through a Millipore Milli-Q ion-exchange system (Bedford, MA). The glassware used was washed in a 50:50 (v/v) ethanol: $1 \mathrm{~mol} \mathrm{dm}^{-3}$ sodium hydroxide bath, followed by a $1 \mathrm{~mol} \mathrm{dm}^{-3}$ nitric acid bath, rinsed copiously with Milli-Q water, and finally dried in an oven.

\subsection{Microorganism maintenance, submerged culture, and media compositions}

T. amestolkiae was kindly provided by the Culture Collection of the Federal University of Amazonas, AM, Brazil. The cultures were reactivated in Czapeck Yeast Extract Agar (CYA) and maintained at $25^{\circ} \mathrm{C}$ for seven days and then stored at $4{ }^{\circ} \mathrm{C}$. The inoculum was plated on potato dextrose agar $\left(39 \mathrm{~g} \mathrm{dm}^{-3}\right)$ supplemented with yeast extract $\left(5 \mathrm{~g} \mathrm{dm}^{-3}\right)$. The inoculum was maintained and stored under the same conditions as detailed above.

For the submerged culture, ten mycelial agar discs ( $8 \mathrm{~mm}$ diameter) from the inoculum were obtained by using a home-made cutter and were transferred into $100 \mathrm{~cm}^{3}$ of the submerged culture medium in $500 \mathrm{~cm}^{3}$ Erlenmeyer flasks. The submerged culture medium was composed of glucose $\left(20 \mathrm{~g} \mathrm{dm}^{-3}\right)$, meat peptone $\left(10 \mathrm{~g} \mathrm{dm}^{-3}\right)$, and meat extract $\left(3 \mathrm{~g} \mathrm{dm}^{-3}\right)$. The $\mathrm{pH}$ of the medium was adjusted to 7.0 with $\mathrm{NaOH}\left(1 \mathrm{~mol} \mathrm{dm}^{-3}\right)$, and the medium was then autoclaved at $121{ }^{\circ} \mathrm{C}$ for $15 \mathrm{~min}$. The submerged culture was incubated in an orbital shaker at $30{ }^{\circ} \mathrm{C}$ at $150 \mathrm{rpm}$ for seven days. At the end of the fermentation process, the fermented broth was filtered using a filter paper $\left(80 \mathrm{~g} \mathrm{~m}^{-2}\right.$; Whatman, UK). The supernatant containing the red colorants was frozen and stored in an ultra-freezer at $-80^{\circ} \mathrm{C}$ for later use in the determination of the binodal curves using the fermented broth and for the partitioning studies.

\subsection{Determination of the binodal curves}

The binodal curves for the systems composed of Triton X-114 (1.0, 3.0, 5.0, 7.0, 9.0, 11.0, 13.0, and $15.0 \mathrm{wt} \%)+\mathrm{IL}\left(\left[\mathrm{C}_{12} \mathrm{mim}\right] \mathrm{Cl}\right.$, $\left[\mathrm{C}_{10} \mathrm{mim}\right] \mathrm{Cl}$, or $\left[\mathrm{C}_{8} \mathrm{mim}\right] \mathrm{Cl}$ at $0.1,0.3$, and $\left.0.5 \mathrm{wt} \%\right)+\mathrm{Mcllvaine} \mathrm{pH}$ 6.5 buffer in the presence or absence of the fermented broth were determined by cloud point titration [21]. The phase-forming components were placed in $15 \mathrm{~cm}^{3}$ glass graduated tubes and weighed to a total mass of $5.0 \mathrm{~g}\left( \pm 10^{-4} \mathrm{~g}\right)$. To evaluate the influence of the fermented broth on the binodal curve, we prepared systems using the same compositions but the buffer solution was replaced with $0.9 \mathrm{~cm}^{3}$ of fermented broth. The solutions were homogenized in a rotary mixer (Labroller II, Labnet) at $8 \mathrm{rpm}$ for $1 \mathrm{~h}$ and then transferred to an ultrathermostatic bath (Ethiktechnology, SP, Brazil). Initially, the temperature was reduced to $10( \pm 1)^{\circ} \mathrm{C}$, where the solution exhibited a single, clear phase. The temperature was then raised slowly (in increments of $0.1{ }^{\circ} \mathrm{C}$ ), and the temperature at which the solution first became cloudy, indicating the onset of phase separation, was defined as $T_{c p}$. The procedure was repeated three times for each data point to ensure reproducibility, and the binodal curve was established by plotting the $T_{c p}$ values as a function of the concentration of Triton X-114 (wt\%).

\subsection{Partitioning of colorants using AMTPS}

Different mixture compositions within the two-phase region were selected and used to evaluate the partitioning of the red colorants between the surfactant- and aqueous-rich phases. Thus, in the first screening, AMTPS with different concentrations of Triton X-114 (1.0, 3.0, 5.0, 7.0, 9.0, 11.0, 13.0, and $15.0 \mathrm{wt} \%$ ) combined with $\left[\mathrm{C}_{12} \mathrm{mim}\right] \mathrm{Cl},\left[\mathrm{C}_{10} \mathrm{mim}\right] \mathrm{Cl}$, or $\left[\mathrm{C}_{8} \mathrm{mim}\right] \mathrm{Cl}$ at different concentrations
$(0.1,0.3$, and $0.5 \mathrm{wt} \%)$ were prepared. For a total mass of $5\left( \pm 10^{-4}\right) \mathrm{g}$, Mcllvaine buffer at $\mathrm{pH} 6.5$ and fermented broth containing the red colorants with an absorbance of 2.0 units at $490 \mathrm{~nm}\left(\mathrm{UA}_{490 \mathrm{~nm}}\right)$ were added. The absorbance was standardized for all experiments to minimize the experimental errors. After the preparation of each AMTPS, the mixture was homogenized for $1 \mathrm{~h}$ in the rotary mixer. To achieve complete partitioning equilibrium, each system was then equilibrated for approximately $24 \mathrm{~h}$ in a thermoregulated water bath (Ethiktechnology, SP, Brazil) at a pre-determined temperature. The set temperature of each system was determined according to the binodal curve to obtain a system with a volumetric ratio $\left(V_{R}\right)$ close to 1.0 . The temperatures used are detailed in Table S1 (Supporting Information).

After the first screening, a second set of experiments was performed to assess the influence of the temperature on the partitioning. Thus, systems with different compositions containing $3 \mathrm{wt} \%$ of Triton X-114 and $0.1 \mathrm{wt} \%$ of $\left[\mathrm{C}_{10} \mathrm{mim}\right] \mathrm{Cl}$ were prepared, as described earlier, and settled at three different temperatures, 25,35 , and $45( \pm 1){ }^{\circ} \mathrm{C}$.

In both sets of experiments, after the equilibrium time $(24 \mathrm{~h})$, the two coexisting micellar phases formed were withdrawn separately, and the red colorants in each phase were determined according to the methodology described in Section 2.5.2. All the experiments were performed in triplicate, and the respective standard deviations determined.

\subsection{Analytical methods}

\subsubsection{Electric conductance measurements}

After the separation of each AMTPS, to determine the IL distribution through the phases, the electric conductance $\left( \pm 0.01 \mathrm{mS} \mathrm{cm}^{-1}\right)$ of both co-existing phases was determined at $25( \pm 1)^{\circ} \mathrm{C}$ using a conductivity meter (914 pH/Conductometer, Metrohm). The measurements were performed in triplicate using systems without fermented broth.

\subsubsection{Quantification of natural red colorants}

The concentration of colorants is usually represented by the color value based on the absorbance spectrum. The concentrations of the red colorants in both co-existing phases were estimated by spectrophotometric analysis of the absorbance at $490 \mathrm{~nm}$ using a UV-Vis multiplate reader (EnSpire Multimode Plate Reader, Perkin Elmer). The wavelength was defined according to the maximum absorbance $[32,33]$. The results are expressed in "units of absorbance at $490 \mathrm{~nm}$ " $\left(\mathrm{UA}_{490 \mathrm{~nm}}\right)$.

The partition coefficient of the red colorant, $K_{R C}$, is defined as the ratio of the absorbance of the colorant in the concentrated (micellerich) $\left(C O L_{M R}\right)$ phase to that in the diluted (micelle-poor) $\left(C O L_{M P}\right)$ phase and was calculated using Eq. (1):

$K_{R C}=\frac{C O L_{M R}}{C O L_{M P}}$

The volumetric ratio $\left(V_{R}\right)$ was determined as described by Eq. (2).

$V_{R}=\frac{V o l_{M R}}{V o l_{M P}}$

Here, $V_{R}$ represents the volumetric ratio (the volume of the micelle-rich phase $\left(\operatorname{Vol}_{M R}\right)$ to the volume of the micelle-poor phase $\left.\left(\operatorname{Vol}_{M P}\right)\right)$.

\section{Results and discussion}

\subsection{Effects of the ILs on the binodal curves}

To evaluate the partitioning of the red colorants, in the first stage, the binodal curves for the AMTPS composed of Triton X-114 with $\left[\mathrm{C}_{8} \mathrm{mim}\right] \mathrm{Cl},\left[\mathrm{C}_{10} \mathrm{mim}\right] \mathrm{Cl}$, or $\left[\mathrm{C}_{12} \mathrm{mim}\right] \mathrm{Cl}$ in the presence of fermented broth were determined. These results are presented in Fig. 2. Moreover, considering the stability and high recovery of the red colorants, the 

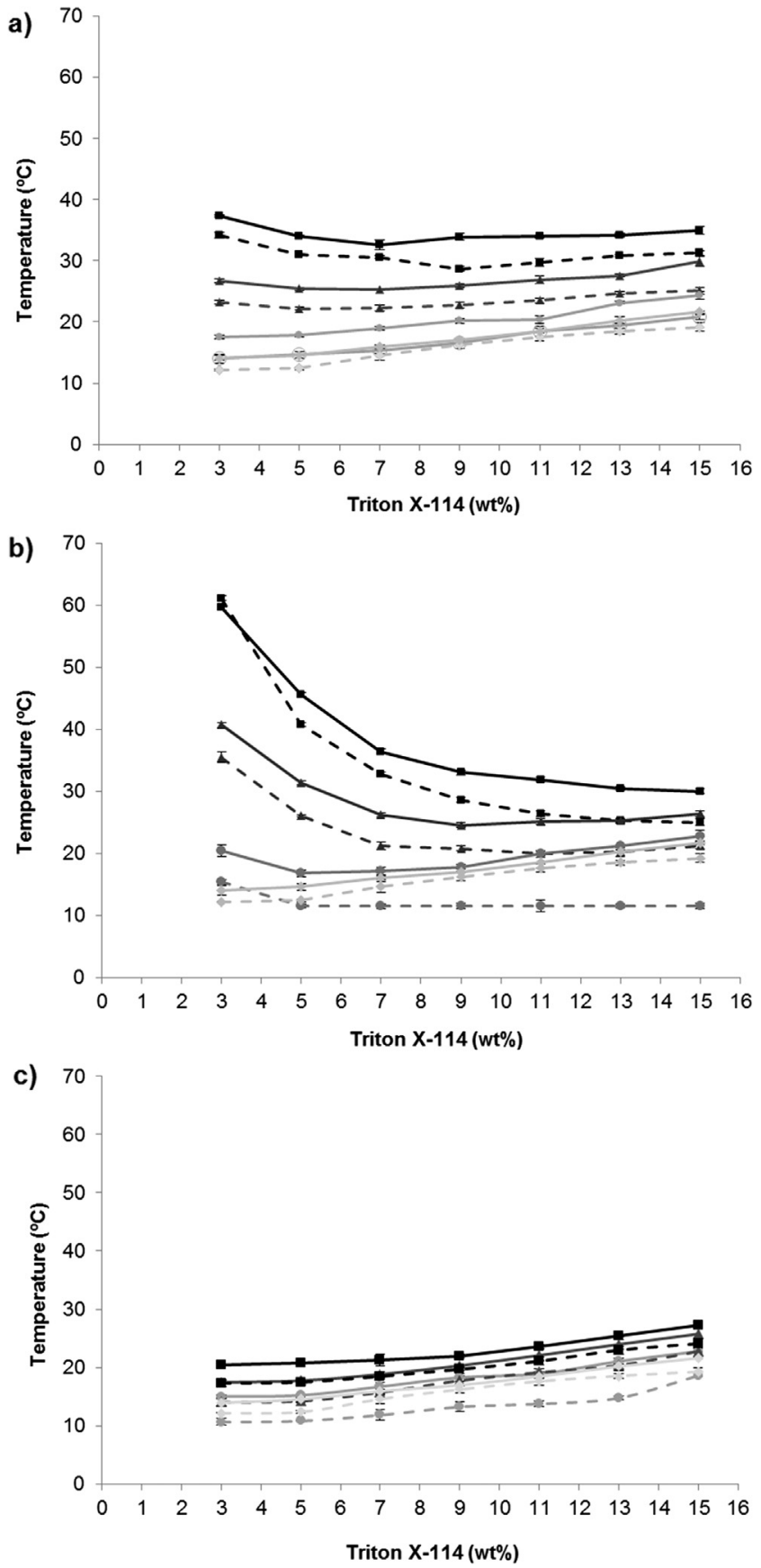

Fig. 2. Binodal curve for the systems composed of Triton X-114 and the following ILs: (a) $\left[\mathrm{C}_{8} \mathrm{mim}\right] \mathrm{Cl}$; (b) $\left[\mathrm{C}_{10} \mathrm{mim}\right] \mathrm{Cl}$; and (c) $\left[\mathrm{C}_{12} \mathrm{mim}\right] \mathrm{Cl}$. The binodal curves for each system were determined in the presence (filled line) and absence (dotted line) of fermented broth. The ILs concentrations are represented by squares ( $0.5 \mathrm{wt} \%)$, triangles $(0.3 \mathrm{wt} \%)$, circles $(0.1 \mathrm{wt} \%)$, and diamonds $(0.0 \mathrm{wt} \%)$. The error bars represent $95 \%$ confidence limits for the measurements.

binodal curves were also determined at a different $\mathrm{pH}$ from that used in the previous work by Vicente and collaborators [13].

The experimental binodal curves presented in Fig. 2 reveal that the addition of the fermented broth affected the phase equilibrium. This is manifested as an increase in the $T_{c p}$ by approximately $12 \%$ for each point for all conditions evaluated. This phenomenon is expected because the fermented broth contains different inorganic salts, residual sugars, and other compounds that can interact with the surfactant molecules, disturbing the micellar structure and consequently reducing the two-phase region equilibrium.

Fig. 2 shows that the $T_{c p}$ raised up with increasing IL concentration.

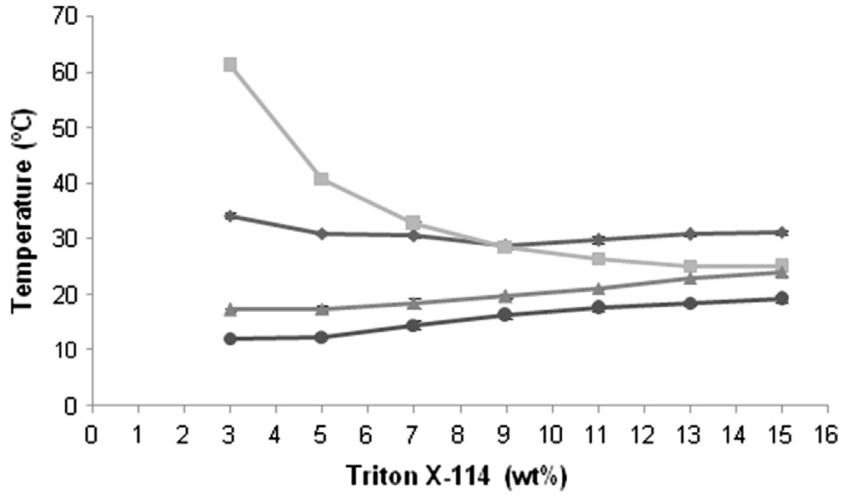

Fig. 3. Binodal curve for systems composed of Triton X-114 and the following ILs, at 0.5 (wt\%): $\left[\mathrm{C}_{12} \mathrm{mim}\right] \mathrm{Cl}$ (triangles), $\left[\mathrm{C}_{10} \mathrm{mim}\right] \mathrm{Cl}$ (squares), and $\left[\mathrm{C}_{8} \mathrm{mim}\right] \mathrm{Cl}$ (diamonds) and without ILs (circles) in the absence of fermented broth. The error bars represent $95 \%$ confidence limits for the measurements.

Bhatt and collaborators [34] documented that the cloud point phenomenon is very sensitive to the presence of additives, and, in this case, when imidazolium-based systems were used, even at very low concentrations, the cloudy region increased. It seems that the addition of the IL changed the CMC, consequently affecting the micelle size [34]. In the present study, the concentration of Triton X-114 used was higher than its CMC in aqueous solution, which is $0.2 \mathrm{mmol} \mathrm{dm}^{-3}\left(20-25^{\circ} \mathrm{C}\right)$ (e.g., $1 \mathrm{wt} \%$ of Triton $\mathrm{X}-114$ corresponds to $18 \mathrm{mmol} \mathrm{dm}^{-3}$ ). Thus, the change in the $T_{c p}$, under the present conditions, is a result of the presence of the ILs, the salts in the Mcllvaine buffer, and the remaining compounds in the fermented broth.

To obtain further insights into the effect of the respective ILs on the binodal curves of the Triton X-114-based AMTPS, it was compared $T_{c p}$ for the systems with $0.5 \mathrm{wt} \%$ of each IL studied, as shown in Fig. 3.

Notably, the presence of the ILs increased the $T_{c p}$. It appears that the increase in the $T_{c p}$ is governed by two different phenomena: (i) the formation of micelles by the surfactants, which is controlled by the tendency of the hydrophobic chain to minimize contact with water, where a more hydrophobic tendency was observed with increasing alkyl chain length; and (ii) the IL charge promotes electrostatic repulsion between the molecules. Thus, in this system, more energy is required to separate the micelle-rich and micelle-poor phases.

The influence of the ILs on the binodal curve was dependent on the concentration of the nonionic surfactant. $\left[\mathrm{C}_{8} \mathrm{mim}\right] \mathrm{Cl}$ caused a lowtemperature deviation, which is expected because this IL has a short alkyl chain. $\left[\mathrm{C}_{10} \mathrm{mim}\right] \mathrm{Cl}$ had the highest $T_{c p}$ of the AMTPS with Triton $\mathrm{X}-114$ contents of 3-7 wt $\%$. Moreover, for the AMTPS composed of $3 \mathrm{wt}$ $\%$ Triton X-114 and $0.5 \mathrm{wt} \%$ of $\left[\mathrm{C}_{8} \mathrm{mim}\right] \mathrm{Cl}$ and $\left[\mathrm{C}_{10} \mathrm{mim}\right] \mathrm{Cl}$, the $T_{c p}$ differed by $\sim 55 \%$. At the highest concentration of Triton X-114, the effect of $\left[\mathrm{C}_{10} \mathrm{mim}\right] \mathrm{Cl}$ on the $T_{c p}$ was less pronounced. The less clear influence of $\left[\mathrm{C}_{12} \mathrm{mim}\right] \mathrm{Cl}$ compared to the other ILs may be due to the strong surfactant-like behavior of this IL, which has a greater effect on the micelle structure.

Jungnickel and collaborators [35] demonstrated that imidazolium ILs having an alkyl chain with at least eight carbons could form micelles. Miskolczy and collaborators [36] also showed that $\left[\mathrm{C}_{8} \mathrm{mim}\right] \mathrm{Cl}$ forms aggregates in aqueous solutions and can form mixed micelles with surfactants, even at low concentrations, because of the more hydrophobic character of the cation [36]. $\left[\mathrm{C}_{12} \mathrm{mim}\right] \mathrm{Cl}$ and $\left[\mathrm{C}_{10} \mathrm{mim}\right] \mathrm{Cl}$ are amphiphilic organic cations (with a long alkyl chain attached to an aromatic ring) associated with a small inorganic anion, and, because of their surfactant-like structures, it is expected that, when these ILs are mixed with aqueous solutions, they will form micelles and exhibit selfassembling behavior [31]. Thakkar and collaborators [37] demonstrated that $\left[\mathrm{C}_{10} \mathrm{mim}\right] \mathrm{Cl}$ penetrates deeper into the Triton X-100 micellar core as a result of the long imidazolium alkyl chain. This phenomenon probably occurs when imidazolium-based ILs with long alkyl 
chains (for example, $\left[\mathrm{C}_{12} \mathrm{mim}\right] \mathrm{Cl}$ ) are used. Considering the literature data, it was expected that all ILs used in this work would act as cosurfactants with Triton X-114.

In all the systems, increasing the concentration of Triton X-114 led to high $T_{c p}$ values. For the systems composed solely of Triton X-114 and buffer, the clouding phenomenon occurred because the increased surfactant concentration induced the formation of a structured water-surfactant system, where the water molecules are bound to more than one micellar system, forming a "barrier" between the micelles and separating them. Consequently, higher temperatures are required to move the water molecules and facilitate the micelle-micelle interactions [23]. However, as demonstrated in the present work and by Vicente et al. [13], the addition of small amounts of ionic surfactants exerts a substantial effect on the cloud point. In work performed by Gu and collaborators [24], the addition of $1 \mathrm{mmol} \mathrm{dm}^{-3}$ sodium dodecyl sulfate (SDS) or cetyltrimethylammonium bromide (CTAB) raised the $T_{c p}$ of a Triton X-114 (1 wt\%) solution by approximately $296 \%$. This is attributed to the formation of mixed micelles. As the ionic surfactant is added to the system, some of these ionic molecules migrate to the nonionic micelles, resulting in their surfaces becoming charged.

To evaluate the dispersion of the ILs in the two macroscopic phases, the conductivities of both co-existing phases were measured. The conductivities of the AMTPS composed of 3 and $15 \mathrm{wt} \%$ Triton X-114 and $0,0.1,0.3$, and $0.5 \mathrm{wt} \%$ IL are compiled in Table S2 (Supporting Information). The temperatures used were the same as those for partitioning of the red colorants, as detailed in Table S1 (Supporting Information). The conductivity data demonstrate that, irrespective of the alkyl chain length of the IL, the micelle-poor phase (top phase) always exhibited the high electrical potential. However, it is important to note that with increasing IL concentration, the electrical conductivity of the micelle-rich phase (bottom phase) increased. This increase shows that the IL tends to stay in the micelle-rich phase, interacting with the nonionic surfactant Triton X-114. The conductivity of the micelle-poor phase remained practically unchanged with the addition of a high concentration of IL to the system, mainly for the systems employing $\left[\mathrm{C}_{8} \mathrm{mim}\right] \mathrm{Cl}$ and $\left[\mathrm{C}_{10} \mathrm{mim}\right] \mathrm{Cl}$. Compared to the results obtained for the system composed of $15 \mathrm{wt} \%$ Triton $\mathrm{X}-114$ using $\left[\mathrm{C}_{10} \mathrm{mim}\right] \mathrm{Cl}$ as an additive, the addition of $0.1 \mathrm{wt} \%$ of IL led to a conductivity of $21.47 \mathrm{mS} \mathrm{cm}^{-1}$ in the top phase and $7.33 \mathrm{mS} \mathrm{cm}^{-1}$ in the bottom phase, whereas, for the addition of $0.5 \mathrm{wt} \% \mathrm{IL}$, the conductivities were 22.67 and $11.27 \mathrm{mS} \mathrm{cm}^{-1}$, respectively, for the top and bottom phases. Thus, it seems that, at high $\mathrm{IL}$ concentrations, $\left[\mathrm{C}_{10} \mathrm{mim}\right] \mathrm{Cl}$ interacts more with Triton X-114, creating mixed micelles, which causes an increase in the conductivity and charge of the system. The system without IL had low conductivity, meaning that some of the IL molecules interact with Triton X-114 while the remaining IL molecules migrate to the top phase. Thus, we believe that some of the IL interacts with Triton X-114, forming mixed micelles, while the rest migrates to the micelle-poor phase. Because the AMTPS are dynamic systems, this phenomenon occurs continuously. To better understand the phenomena occurring in the systems composed of Triton X-114 and imidazolium-based ILs, further experiments to characterize the micelles will be performed (this was not the focus of the present work).

The partitioning behavior of biomolecules is strongly related to the binodal curves of the AMTPS; for many cases, the binodal curve can be easily found in the literature, and, thus, the partitioning experiments can be performed directly. However, as demonstrated in this section, when the aim is to extract a biomolecule from a complex fermented broth, the influence of the fermented media on the binodal curve and the effect of the balance of the interactions in the micelles at equilibrium must be investigated.

\subsection{Extraction of red colorants from fermented broth using AMTPS}

The extraction and purification of different biomolecules using AMTPS require a series of different conditions that can directly

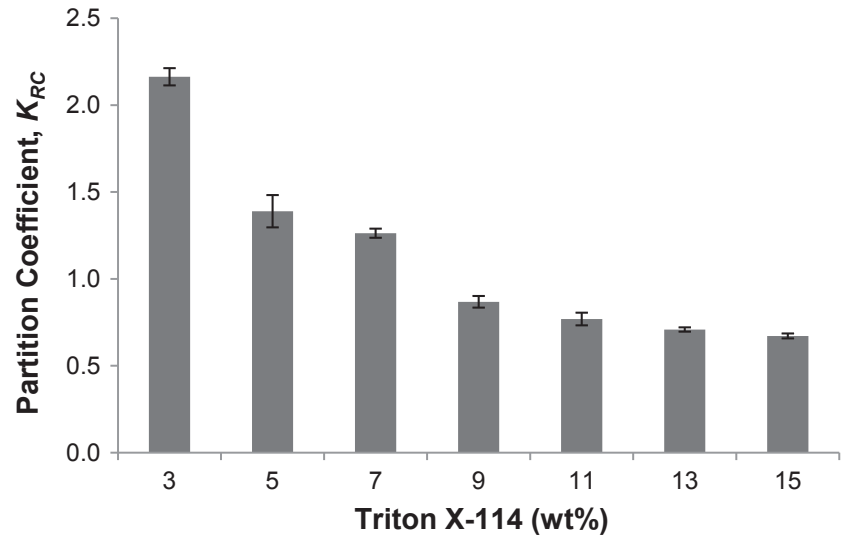

Fig. 4. Partition coefficients of red colorants $\left(K_{R C}\right)$ from the fermented broth produced by T. amestolkiae using AMTPS composed of different concentrations of Triton X-114 (3-15 wt $\%$ ) in Mcllvaine buffer at $\mathrm{pH}$ 6.5. The error bars represent $95 \%$ confidence limits for the measurements.

influence the partitioning of the solute into one of the co-existing phases; these conditions include the chemical composition, temperature, $\mathrm{pH}$, or the addition of specific adjuvants, affinity ligands, or amphiphilic structures [38]. Thus, considering the binodal curves obtained from tests with the fermented broth (Fig. 2), different settling temperatures were defined for each partitioning mixture to ensure that the mixture selected would result in two macroscopic phases (the temperatures are presented in Table S1; Supporting Information).

\subsubsection{Effect of Triton $X-114$ concentration}

An initial screening was performed to evaluate the partitioning of the red colorants by using AMTPS with different concentrations of Triton X-114 (3-15 wt\%) and Mcllvaine buffer at $\mathrm{pH}$ 6.5, without the addition of any IL; the results are compiled in Fig. 4.

Analysis of the data in Fig. 4 demonstrates that the colorants partitioned into the micelle-rich phase $\left(K_{R C}>1.0\right)$ for the systems with low concentrations of Triton X-114 (3-7 wt\%), whereas, at high surfactant concentrations (9-15 wt\%), the red colorants were equally distributed throughout both phases ( $K_{R C}$ close to 1$)$. One hypothesis for this behavior is that an increase in the concentration of Triton X-114 increases the hydrophobicity of the bottom phase (micelle-rich phase), thereby increasing the affinity of the red colorants for the top phase (micelle-poor phase).

Although the structure of the colorant is unknown, the previous results of our group [39] showed that red colorants in the fermented broth of T. amestolkiae have negative octanol/water partition coefficient at $\mathrm{pH}$ 6.5, characteristic of hydrophilic biocompounds. It is important to clarify that, in the fermented broth, the colorants may be linked with other more hydrophilic compounds, and the colorant itself is not necessarily a hydrophilic molecule. Thus, it has been observed that when the relative hydrophobicity of the micelle-rich phase was increased (i.e., a higher Triton X-114 concentration), the colorant began to migrate to the more hydrophilic phase (micelle-poor phase). However, considering that both co-existing phases are water-rich environments, other types of interactions can influence the partitioning of the molecules, such as electrostatic repulsive interactions and volume exclusion effects. Thus, it is quite clear that, at low concentrations of Triton X-114, the mechanism governing the migration of the red colorants is not related to their hydrophilicities (hydrogen bonding type interactions) since the solute is preferentially partitioned into the water-poor phase (despite its hydrophilic characteristics). The AMTPS with only $3 \mathrm{wt} \%$ Triton X-114 showed the highest ability to concentrate the red colorant, having a $K_{R C}$ of 2.16 . This system was 3 -fold more efficient in the extraction of the red colorant than the system with $15 \mathrm{wt}$ $\%$ Triton X-114. For the system with $3 \mathrm{wt} \%$ Triton X-114, the 
conductivity of the top phase (micelle-poor phase) of the system was twice as high as that of the micelle-rich phase.

Furthermore, the increased partitioning of the red colorants into the micelle poor-phase (top phase), observed at high concentrations of Triton X-114, may also be influenced by some volume exclusion effects or by an increase in the relative hydrophobicity of this phase. In terms of the volume exclusion effects, Triton X-114 has a high molecular weight $\left(536 \mathrm{~g} \mathrm{~mol}^{-1}\right)$, and, with increasing surfactant concentration, a large number of micelles will be created. With more micelles in the micelle-rich phase, the space available for the red colorant molecules decreases (making it more difficult for them to remain in this phase), and the molecules will be partitioned into the surfactant-poor-phase, leading to a decrease in the $K_{R C}$ as the concentration of Triton X-114 increases.

There are no documented studies of the recovery of natural colorants using AMTPS; thus, this is the first attempt to evaluate these systems in the presence of ILs for the extraction of colorants from fermented broth.

\subsubsection{Effects of different ILs}

After the first screening to assess the influence of the content of Triton X-114 on the extraction of the red colorants, three series of AMTPS were prepared by combining Triton X-114, Mcllvaine buffer at $\mathrm{pH}$ 6.5, and the following imidazolium-based ILs as adjuvants: $\left[\mathrm{C}_{8} \mathrm{mim}\right] \mathrm{Cl},\left[\mathrm{C}_{10} \mathrm{mim}\right] \mathrm{Cl}$, and $\left[\mathrm{C}_{12} \mathrm{mim}\right] \mathrm{Cl}$. The mixtures were prepared, and the extraction of the red colorants was performed at pre-specified temperatures (Table S1, Supporting Information). The results obtained for each series of partitioning studies are presented Fig. 5.

Fig. 5 shows that the red colorant was preferentially partitioned into the micelle-rich phase (more hydrophobic). The AMTPS using ILs as surfactants showed a similar trend to that of the systems composed solely of Triton X-114 (Fig. 4), where, as the concentration of the surfactant increased, the $K_{R C}$ decreased. However, the presence of the ILs promoted a high partitioning of the red colorants into the micelle-rich phase when low concentrations of Triton X-114 were used. To evaluate if attractive electrostatic interactions were operative in the system, the zeta potential of both coexisting phases were measured for all systems, as well as for the red colorants dissolved in pH 6.5 buffer (results not shown). The presence of the ILs induced the formation of positively charged mixed micelles, resulting in the attraction of the red colorants (negatively charged at $\mathrm{pH}$ 6.5). This phenomenon was particularly pronounced at low concentrations of Triton X-114, where the relative hydrophobicity of the micelle-rich phase is reduced, increasing the electrostatic interactions between the colorants and charged micelles. However, with an increase in the concentration of Triton X-114 and the ILs, the micelle-rich phase became quite hydrophobic. Consequently, it is probable that the electrostatic interactions between the red colorant and the ILs were not sufficient strong to keep the target biomolecule in the micelle-rich phase and the red colorants start to migrate to the more hydrophilic environment of the micelle-poor phase.

The AMTPS composed of Triton X-114 (3 wt\%, which was the lowest concentration of surfactant) and 0.1 and $0.3 \mathrm{wt} \%$ of $\left[\mathrm{C}_{12} \mathrm{mim}\right] \mathrm{Cl}$ had respective $K_{R C}$ values of 4.9 and 5.1. The systems composed of Triton $\mathrm{X}-114(3 \mathrm{wt} \%)$ and $0.1 \mathrm{wt} \%$ of $\left[\mathrm{C}_{10} \mathrm{mim}\right] \mathrm{Cl}$ gave a rise to the highest $K_{R C}$ (6.1), whereas for the systems with the same concentration of Triton X-114 and $0.1 \mathrm{wt} \%\left[\mathrm{C}_{8} \mathrm{mim}\right] \mathrm{Cl}$, the $K_{R C}$ was 4.9 . The partitioning aptitude of the $\left[\mathrm{C}_{10} \mathrm{mim}\right] \mathrm{Cl}$-based AMTPS with $3 \mathrm{wt} \%$ Triton $\mathrm{X}$ 114 and $0.5 \mathrm{wt} \%$ IL was not assessed because the $T_{c p}$ was higher than $80{ }^{\circ} \mathrm{C}$.

The series of results shown in Fig. 5 demonstrated that, in general, the addition of small amounts of IL to the system improved the partitioning of the red colorants produced by $T$. amestolkiae into the micellerich phase. Furthermore, the migration of the colorants was favored in AMTPS at low surfactant concentrations, where the number of micelles in Triton X-114 was lower, probably because of electrostatic attraction between the colorants and the ILs. Although both main partitioning
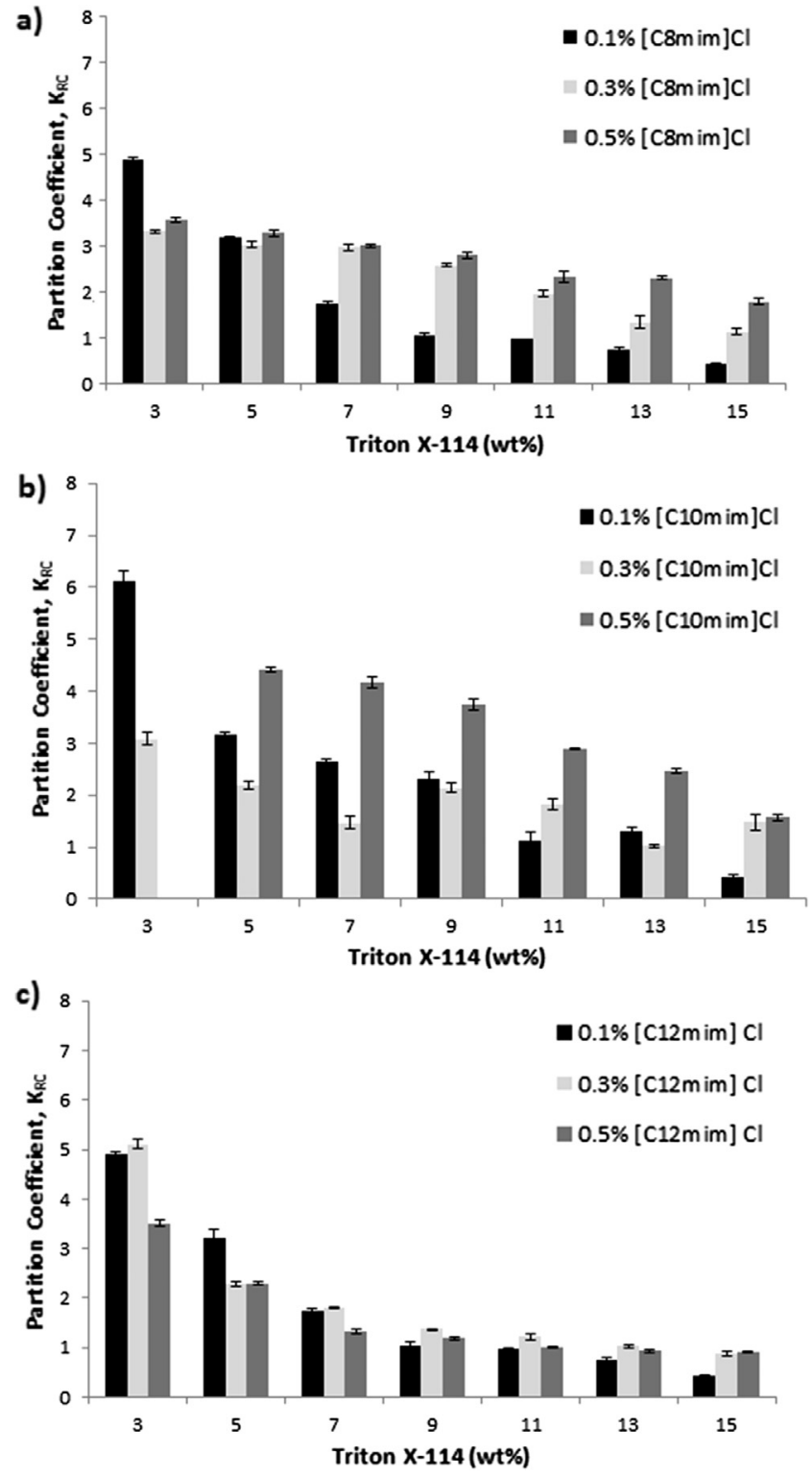

Fig. 5. Partition coefficients of red colorants $\left(K_{R C}\right)$ from the fermented broth produced by T. amestolkiae using AMTPS composed of different concentrations of Triton X-114 (3-15 wt\%), Mcllvaine buffer at $\mathrm{pH} 6.5$ and different concentrations $(0.1,0.3$, and $0.5 \mathrm{wt}$ $\%)$ of the following ILs as co-surfactants: a) $\left[\mathrm{C}_{8} \mathrm{mim}\right] \mathrm{Cl}$; b) $\left[\mathrm{C}_{10} \mathrm{mim}\right] \mathrm{Cl}$; and c) $\left[\mathrm{C}_{12} \mathrm{mim}\right] \mathrm{Cl}$. The error bars represent $95 \%$ confidence limits for the measurements.

trends were noticed for all the systems, from the data of each IL-based AMTPS, three distinct trends are clearly observed: (i) there is an increase in the $K_{R C}$ with 0.3 and $0.5 \mathrm{wt} \%$ of $\left[\mathrm{C}_{8} \mathrm{mim}\right] \mathrm{Cl}$ when the Triton $\mathrm{X}-114$ concentration was greater than $5 \mathrm{wt} \%$ (Fig. $5 \mathrm{a}$ ); (ii) it occurs an increase in the $K_{R C}$ with the addition of $0.5 \mathrm{wt} \%$ [C $\left.\mathrm{C}_{10} \mathrm{mim}\right] \mathrm{Cl}$ (Fig. $5 \mathrm{~b}$ ); and (iii) the addition of $\left[\mathrm{C}_{12} \mathrm{mim}\right] \mathrm{Cl}$ has a negligible effect on the $K_{R C}$ (Fig. 5c). These trends can be easily understood based on the balance of the hydrophobic and electrostatic interactions and the volume exclusion effects between the colorant molecules and the micelles induced by the ILs, as well as in terms of the change in the size and structure of the micelles. Although further structural studies are required to fully reveal the partitioning mechanisms at the molecular level, for imidazoliumbased ILs with a long alkyl chain co-surfactants, such as $\left[\mathrm{C}_{12} \mathrm{mim}\right] \mathrm{Cl}$, the partitioning of the solutes occurs via an entirely different driving mechanism than that of the shorter IL $\left(\left[\mathrm{C}_{8} \mathrm{mim}\right] \mathrm{Cl}\right)$. The use of surfactant-like ILs promotes the formation of mixed micelles that act as traditional surfactant AMTPS, where the concentration of the micelles 
Table 1

Partition coefficient of red colorants $\left(K_{R C}\right)$ and volumetric ratio $\left(V_{R}\right)$ for AMTPS composed of $3 \mathrm{wt} \%$ Triton $\mathrm{X}-114$ and $0.1 \mathrm{wt} \%\left[\mathrm{C}_{10} \mathrm{mim}\right] \mathrm{Cl}$ at 25,35 and $45^{\circ} \mathrm{C}$.

\begin{tabular}{lll}
\hline Temperature $\left({ }^{\circ} \mathrm{C}\right)^{\mathrm{a}}$ & $K_{R C}{ }^{\mathrm{a}}$ & $V_{R}{ }^{*}$ \\
\hline 25 & $6.56 \pm 0.34^{\mathrm{a}}$ & $1.27 \pm 0.00^{\mathrm{a}}$ \\
35 & $20.29 \pm 0.96^{\mathrm{b}}$ & $0.25 \pm 0.00^{\mathrm{b}}$ \\
45 & $24.40 \pm 0.74^{\mathrm{c}}$ & $0.09 \pm 0.00^{\mathrm{c}}$ \\
\hline
\end{tabular}

a Standard uncertainty $\mathrm{u}$ are $\mathrm{u}(\mathrm{T})= \pm 1{ }^{\circ} \mathrm{C}$; $\mathrm{u}($ Abs $)=0.001 \mathrm{UA}$.

* The error represents $95 \%$ confidence limits for the measurements. Values that share the same letter in a column are not significantly different at the $5 \%$ level.

is the main factor influencing the partitioning. On the other hand, with the use of shorter ILs as the co-surfactants, the charge distributions of the ions and their ability to establish specific interactions with the solutes become the main factor governing the migration.

\subsubsection{Effect of temperature}

The temperature is a major factor in the partitioning of several solutes, and, considering that different AMTPs exhibit different $T_{c p}$ values, it is crucial to understand how the temperature affects the extraction of the red colorant produced by T. amestolkiae. Thus, in this section, the influence of temperature on the partitioning of the red colorant is evaluated. For this purpose, three different AMTPS composed of $3 \mathrm{wt} \%$ Triton X-114 and $0.1 \mathrm{wt} \%\left[\mathrm{C}_{10} \mathrm{mim}\right] \mathrm{Cl}$ in Mcllvaine $\mathrm{pH} 6.5$ buffer (the system that exhibited the highest partitioning ability in the previous screening) were prepared and used to separate the red colorants at three temperatures $\left(25,35\right.$, and $\left.45^{\circ} \mathrm{C}\right)$. The $K_{R C}$ and the $V_{R}$ from three independent assays are compared in Table 1.

The results clearly show that the temperature has a significant effect on the partitioning of the red colorant produced by T. amestolkiae. A $10{ }^{\circ} \mathrm{C}$ rise in the settling temperature of the AMTPS greatly increased the $K_{R C}$. A comparison of the partitioning ability of the AMTPS (settled at $\left.45{ }^{\circ} \mathrm{C}\right)$ at the lowest $\left(25^{\circ} \mathrm{C}\right)$ and highest $\left(45^{\circ} \mathrm{C}\right)$ temperatures showed a 3.8 -fold increase at the highest temperature. Furthermore, it is important to point out that most of the systems used in this work (previous sections) have $V_{R}$ values close to 1 (Table S3, Supporting Information). The temperature increase caused a decrease in the $V_{R}$ of the Triton X$114 /\left[\mathrm{C}_{10} \mathrm{mim}\right] \mathrm{Cl}$-based AMTPS (From $V_{R 25^{\circ} \mathrm{C}}=1.27$ to $V_{R 45^{\circ} \mathrm{C}}=0.09$ ). Thus, at higher temperatures, the reduction in the volume of the bottom phase caused an increase in the concentration of the red colorants in the surfactant-rich phase, and, consequently, the corresponding $K_{R C}$ increased. The temperature strongly affects the entropy of systems; thus, because the AMTPS are dynamic systems, with increasing temperature, the entropy of the systems increased, and a large amount of energy was provided to the system. Consequently, the electrostatic interactions between the charged red colorants and mixed micelles were enhanced (high $K_{R C}$ values).

In summary, the results obtained in this work, after the temperature adjustment, are quite promising and support the use of these AMTPS as effective alternatives for the extraction of red colorants from T. amestolkiae fermented broth. Comparison with previous studies shows that these systems exhibit a strong ability to concentrate the red colorants relative to previous systems using colloidal gas aphrons and CTAB as surfactants [39], systems for which the maximum partitioning coefficient was 5.5. In the cited work, the authors used a positively charged (CTAB) and negatively charged (SDS) surfactants, and the colorant was partitioned into the CTAB-rich phase, possibly because of the electrostatic attraction between the negatively charged solutes and the positively charged CTAB.

\section{Conclusion}

The use of ILs from the imidazolium family as a co-surfactant in AMTPS was evaluated for the extraction of the red colorant from the fermented broth of T. amestolkiae. The binodal curves for the AMTPS composed of Triton X-114 with $\left[\mathrm{C}_{8} \mathrm{mim}\right] \mathrm{Cl},\left[\mathrm{C}_{10} \mathrm{mim}\right] \mathrm{Cl}$, or $\left[\mathrm{C}_{12} \mathrm{mim}\right] \mathrm{Cl}$ in the presence and absence of fermented broth were determined. The addition of ILs and the presence of fermented broth induced an increase on the $T_{C P}$. The addition of ILs affected the $T_{C P}$ by the diminishing of the contact with water with an increase in the alkyl chain length and due to electrostatic repulsions between charged molecules. The increase of the $T_{C P}$ by presence of the fermented broth was caused by the presence of residual contaminants (salts, sugar) of the broth and their interactions with surfactant molecules.

The red colorant partitioning aptitude of each AMTPS was evaluated, being the colorant preferentially partitioned into the micelle-rich phase with low Triton X-114 concentrations and equally distributed between both phases in equilibrium at high surfactant concentrations. The best result was obtained for the system composed with $3 \mathrm{wt} \%$ Triton X-114. However, the addition of 0.1 or $0.3 \mathrm{wt} \%$ of the $\left[\mathrm{C}_{12} \mathrm{mim}\right] \mathrm{Cl}$ or $\left[\mathrm{C}_{10} \mathrm{mim}\right] \mathrm{Cl}$ increased the $K_{R C}$ molecule by approximately 2.3-fold when compared to the AMTPS without ILs. The addition of low ILs' concentrations favored the red colorants partitioning into the micelle-rich phase when low concentrations of Triton X-114. It seems that the formation of ILs-Triton X-114 mixed micelles occurred, and some favorable electrostatic attraction between the colorants and the ILs enhancing the migration of the colorants into the micelles. Although the partitioning mechanisms are not fully understood the set of the results obtained in this work clearly demonstrates that the addition of small concentrations of $\left[\mathrm{C}_{12} \mathrm{mim}\right] \mathrm{Cl}$ or $\left[\mathrm{C}_{10} \mathrm{mim}\right] \mathrm{Cl}$ to the AMTPS improved the partitioning of the target molecule. Furthermore, the temperature effect on the partitioning was also addressed, where a $10{ }^{\circ} \mathrm{C}$ rise in the settling temperature of the AMTPS enhances greatly the values of the $K_{R C}$, highlighting the importance of this parameter in the partitioning of the molecules when used AMTPS.

\section{Acknowledgements}

The authors are grateful to Prof. Dr. Edwil Aparecida de Lucca Gattas, Prof. Dr. Celia Maria de Sylos, Prof. Dr. André Gonzaga dos Santos and Prof. Dr. Adriano Mondini for allowing us to carry out our experiments in their laboratory. Jorge F.B. Pereira acknowledges the funding from FAPESP - Brazil (2014/16424-7).

\section{Funding}

This work was supported by the São Paulo Research Foundation (FAPESP) - Brazil [Grant Nos. 2014/01580-3, 2015/04751-6 and 2016/01076-9] and by CNPq - Brazil [Grant No. 443984/2014-0].

\section{Appendix A. Supplementary material}

Supplementary data associated with this article can be found, in the online version, at http://dx.doi.org/10.1016/j.seppur.2017.07.056.

\section{References}

[1] K. Malik, J. Tokkas, S. Goyal, Microbial pigments: a review, Int. J. Microb. Resource Technol. 163 (2012) 361-365.

[2] M. Shahid, S. Islam, F. Mohammad, Recent advancements in natural dye applications: a review, J. Clean. Prod. 53 (2013) 310-331.

[3] C. Gerardi, N. Tommasi, C. Albano, F. Blando, L. Rescio, E. Pinthus, G. Mita, Prunus mahaleb L. fruit extracts: a novel source for natural food pigments, Eur. Food Res. Technol. 241 (2015) 683-695.

[4] A. Bouzidi, N. Baaka, N. Salem, M.F. Mhenni, Z. Mighri, Limoniastrum monopetalum stems as a new source of natural colorant for dyeing wool frabrics, Fyber Polym. 17 (2016) 1256-1261.

[5] F.A.E. Torres, B.R. Zaccarim, L.C.L. Novaes, A.F. Jozala, C.A. dos Santos, M.F.S. Teixeira, V.C.S. Ebinuma, Natural colorants from filamentous fungi, Appl. Microbiol. Biotechnol. 100 (2016) 2511-2521.

[6] L. Dufossé, M. Fouillaud, Y. Caro, S.A.S. Mapari, N. Sutthiwong, Filamentous fungi are large-scale producers of pigments and colorants for the food industry, Curr. Opin. Biotechnol. 26 (2014) 56-61.

[7] J.C. Frisvad, N. Yilmaz, U. Thrane, K.B. Rasmussen, J. Houbraken, R.A. Samson, Talaromyces atroroseus, a new species efficiently producing industrially relevant red 
pigments, PLOS One. 8 (2013).

[8] F. Delgado-Vargas, A.R. Jiménez, O. Paredes-López, Natural pigments: carotenoids, anthocyanins, and betalains-characteristics, biosynthesis, processing, and stability, Crit. Rev. Food. Sci. Nutr. 40 (2010) 173-289.

[9] N. Rombaut, A.S. Tixier, A. Billy, F. Chemat, Green extraction processes of natural products as tools for biorefinery, Biofuels Bioprod. Biored. 8 (2014) 530-544.

[10] V.C. Santos, F.A. Hasmann, A. Converti, A. Pessoa Jr., Liquid-liquid extraction by mixed micellar systems: a new approach for clavulanic acid recovery from fermented broth, Biochem. Eng. J. 56 (2011) 75-83.

[11] M. Dermiki, M.H. Gordon, P. Jauregi, Recovery of astaxanthin using colloidal gas aphrons (CGA): a mechanistic study, Sep. Purif. Technol. 65 (2009) 54-64.

[12] M. Amid, M.Y.A. Manap, M. Shuhaimi, Purification of a novel protease enzyme from kesinai plant (Streblus asper) leaves using a surfactant-salt aqueous micellar two-phase system: a potential low cost source of enzyme and purification method, Eur. Food Res. Technol. 237 (2013) 601-608.

[13] F.A. Vicente, L.P. Malpiedi, F.A. Silva, A. Pessoa Jr., J.A.P. Coutinho, S.P.M. Ventura, Design of novel aqueous micellar two-phase system using ionic liquids as co-surfactants for the selective extraction of (bio)molecules, Sep. Purif. Technol. 135 (2014) 259-267.

[14] M.G. Freire, A.F.N. Cláudio, J.M.M. Araújo, J.A.P. Coutinho, I.M. Marrucho, J.N.C. Lopes, L.P.N. Rebelo, Aqueous biphasic systems: a boost brought about by using ionic liquids, Chem. Soc. Rev. 41 (2012) 4966-4995.

[15] J.F.B. Pereira, L.P.N. Rebelo, R.D. Rogers, J.A.P. Coutinho, M.G. Freire, Combining ionic liquids and polyethylene glycols to boost the hydrophobic-hydrophilic range of aqueous biphasic systems, Phys. Chem. Chem. Phys. 15 (2013) 19580-19583.

[16] M.S.C. Silva, V.C. Santos-Ebinuma, A.M. Lopesa, C.O. Rangel-Yagui, Dextran sulfate/Triton X two-phase micellar systems as an alternative first purification step for clavulanic acid, Fluid Phase Equilibr. 399 (2015) 80-86.

[17] C.W. Ooi, C.P. Tan, S.L. Hii, A. Ariff, S. Ibrahim, T.C. Ling, Primary recovery of lipase derived from Burkholderia sp. ST8 with aqueous micellar two-phase system, Process Biochem. 40 (2011) 1847-1852.

[18] C.B. Ojeda, F.S. Rojas, Separation and preconcentration by cloud point extraction procedures for determination of ions: recent trends and applications, Microchim. Acta. 177 (2012) 1-21.

[19] V.C. Santos-Ebinuma, A.M. Lopes, A. Converti, A. Pessoa Jr., C. Oliveira, RangelYagui, Behavior of Triton X-114 cloud point in the presence of inorganic electrolytes, Fluid Phase Equilibr. 360 (2013) 435-438.

[20] P.L. Show, T.C. Ling, J.C.W. Lan, B.T. Tey, R.N. Ramanan, S.T. Yong, C.W. Ooi, Review of microbial lipase purification using aqueous two-phase systems, Curr. Org. Chem. 19 (2015) 19-29.

[21] C.L. Liu, Y.J. Nikas, D. Blankschtein, Novel bioseparation using two-phase aqueous micellar systems, Biotechnol. Bioeng. 52 (1996) 185-192.

[22] L.-S. Hao, P. Hu, Y.-Q. Nan, Salt effect on the rheological properties of the aqueous mixed cationic and anionic surfactant systems, Colloids Surf. A. 361 (2010) 187-195.

[23] L. Koshy, A.H. Saiyad, A.K. Rakshit, The effects of various foreign substances on the cloud point of Triton X 100 and Triton X 114, Colloid Polym. Sci. 274 (1996)
$582-587$.

[24] T. Gu, P.A. Galera-Gómez, Clouding of Triton X-114: The effect of added electrolytes on the cloud point of Triton X-114 in the presence of ionic surfactants, Colloids Surf. A. 104 (1995) 307-312.

[25] K.E. Gutwoski, G.A. Broker, H.D. Willauer, J.G. Huddleston, R.P. Swatloski, J.D. Holbrey, R.D. Rogers, Controlling the aqueous miscibility of ionic liquids: aqueous biphasic systems of water-miscible ionic liquids and water-structuring salts for recycle, metathesis and separations, J. Am. Chem. Soc. 125 (2003) 6632-6633.

[26] L. Vidal, M.L. Riekkola, A. Canals, Ionic liquid-modified materials for solid-phase extraction and separation: a review, Anal. Chim Acta. 715 (2012) 19-41.

[27] A.E. Somers, P.C. Howlett, D.R. MacFarlane, M. Forsyth, A review of ionic liquid lubricants, Lubricants. 1 (2013) 3-21.

[28] I. Rodríguez-Escontrela, I. Rodríguez-Palmeiro, O. Rodríguez, A. Arce, a. Soto, Characterization and phase behavior of the surfactant ionic liquid tributylmethylphosphonium dodecylsulfate for enhanced oil recovery, Fluid Phase Equilibr. 417 (2016) 87e95.

[29] J. Dupont, On the solid, liquid and solution structural organization of imidazolium ionic liquids, J. Braz. Chem. Soc. 15 (2004) 341-350.

[30] G.S. Fonseca, G. Machado, S.R. Teixeira, G.H. Fecher, J. Morais, M.C.M. Alves, J. Dupont, Synthesis and characterization of catalytic iridium nanoparticles in imidazolium ionic liquids, J. Colloid Interf. Sci. 201 (2006) 193-204.

[31] A. Moderassi, H. Sifaoui, M. Mielcarz, U. Domanska, M. Rogalski, Influence of the molecular structure on the aggregation of imidazolium ionic liquids in aqueous solutions, Colloids Surf., A. 302 (2007) 181-185.

[32] M.R. Johns, D.M. Stuart, Production of pigments by Monascus purpureus in solid culture, J. Ind. Microbiol. 8 (1991) 23-38.

[33] V.C. Santos-Ebinuma, I.C. Roberto, M.F.S. Teixeira, A. Pessoa Jr., Improvement of submerged culture conditions to produce colorants by Penicillium purpurogenum, Braz. J. Microbiol. 45 (2014) 731-742.

[34] D. Bhatt, K.C. Maheria, J. Parikh, Studies on surfactant-ionic liquid interaction on clouding behavior and evaluation of thermodynamic parameters, J. Surfact. Deterf. 16 (2013) 547-557.

[35] C. Jungnickel, J. Luczak, J. Ranke, J.F. Fernández, A. Müller, J. Thöming, Micelle formation of imidazolium ionic liquids in aqueous solution, Coll. Surf. A Physicochem Eng. Asp. 316 (2008) 278-284.

[36] Z. Miskolczy, K. Sebok-Nagy, L. Biczok, S. Gokturk, Aggregation and micelle formation of ionic liquids in aqueous solution, Chem. Phys. Lett. 400 (2004) 296-300.

[37] K. Thakkar, V. Patel, D. Ray, H. Pal, V.K. Aswal, P. Bahadur, Interaction of imidazolium based ionic liquids with Triton X-100 micelles: investigating the role of the counter ion and chain length, RSC Adv. 6 (2016) 36314-36326.

[38] Y. Liu, Y. Zhang, X. Wu, X. Yan, Effect of excluded-volume and hydrophobic interactions on the partition of proteins in aqueous micellar two-phase systems composed of polymer and nonionic surfactant, Fluid Phase Equilibr. 429 (2016) 1-8.

[39] V.C. Santos-Ebinuma, M.F.S. Teixeira, A. Pessoa Jr., P. Jauregi, Separation of natural colorants from the fermented broth of filamentous fungi using colloidal gas aphron, Sep. Purif. Technol. 163 (2016) 100-108. 